\title{
Geometric phases and Wannier functions of Bloch electrons in 1-dimension
}

\author{
Joydeep Bhattacharjee and Umesh V Waghmare \\ Theoretical Sciences Unit, \\ Jawaharlal Nehru Centre for Advanced Scientific Research, \\ Jakkur PO, Bangalore 560 064, India
}

\begin{abstract}
We present a formal expression for Wannier functions of composite bands of 1-D Bloch electrons in terms of parallel-transported Bloch functions and their non-Abelian geometric phases. Spatial decay properties of these Wannier functions are studied in the case of simple bands of 1-D model insulator and metal. Within first-principles density functional theory, we illustrate the formalism through the construction of Wannier functions of polyethylene and polyacetylene.
\end{abstract}

PACS numbers: 71.15.-m 71.20.-b 71.23.An

Since the introduction in 1937, Wannier functions (WF) 1] have been conceptually interesting and useful in studies of electronic properties of materials. They highlight the atomic orbital character of electrons and chemical bonding 2] and appear naturally in the symmetry and analytical properties of energy bands in solids 3, 44. They form an excellent basis set for use in the construction of first-principles model Hamiltonians in studies of phase transitions [5, 6]. There is growing interest in WFs as they provide a localized basis in real space for the description of electronic states and are useful in the implementation of computationally efficient "linear scaling" algorithms [7, 8].

WFs are obtained as a unitary transformation of Bloch functions 11. A relationship between the first moment of squared WFs (density) and geometric phases [9] of Bloch electron was uncovered in the context of adiabatic dynamics of Bloch electron 10 and later in the modern theory of polarization 11, 12]. WFs are not unique due to the freedom in choice of phases accompanying Bloch functions, but they are desired to be highly localized for practical applications. The second moment (cumulant) of squared WFs, a measure of their spatial range, is used as an objective function for minimization in the variational procedure to construct WFs developed by Marzari and Vanderbilt [13].

In this paper, we focus on the solution of WF problem for systems periodic in 1-D, and comment on its relation and extension to higher dimensions. We use Bloch functions parallel transported along the Bloch vector $\mathbf{k}$ in the Brillouin zone as a starting point in the construction of WFs. As these are not periodic in reciprocal space, we present an explicit form for a unitary transformation to construct a set that is periodic as a function of $\mathbf{k}$. For the 1-D case, we show that the WFs obtained as a Fourier transform of this set are maximally localized.

Starting with a subspace $S_{k_{0}}$ of $N$ energy eigenfunctions at $k_{0}$, we use parallel transport to obtain Bloch functions $e^{i k x} u_{k n}^{\|}(x)$ for $k \in\left(k_{0}, k_{0}+\frac{2 \pi}{a}\right)$, a being the lattice constant and $n$ being an index for electronic states. This is accomplished using $\mid \frac{\partial u_{k n}}{\partial k}>$ obtained routinely within the parallel transport gauge:

$$
\left\langle u_{k m}^{\|} \mid \frac{\partial u_{k n}^{\|}}{\partial k}\right\rangle=0
$$

in density functional theory linear response 14, 15] (or k.p perturbation theory) calculations. We use second-order Runge-Kutta procedure 16] to translate from $u_{k n}^{\|}(x)$ to $u_{k+\Delta k n}^{\|}(x)$. Geometric phases, generalized for degenerate states 17] and paths closed within a gauge transformation [10], form an $N \times N$ Hermitian matrix $\Gamma$ and are given by:

$$
\left[e^{i \Gamma}\right]_{m n}=\left\langle u_{m k_{0}}^{\|}\left|\exp \left(i \frac{2 \pi x}{a}\right)\right| u_{n k_{0}+\frac{2 \pi}{a}}^{\|}\right\rangle
$$

The electronic part of the macroscopic polarization 11 $P$ is readily given by $\frac{2 \pi e}{a} \operatorname{Tr}(\Gamma)$.

Non-diagonal form of the matrix $\Gamma$, in general, indicates that a starting wavefunction at $k_{0}$ mixes with other members of the subspace $S_{k_{0}}$ as $k$ is transported to $k_{0}+\frac{2 \pi}{a}$, amounting to a unitary transformation of the subspace $S_{k_{0}}$. Diagonalization of $\Gamma$ and corresponding transformation of Bloch functions in $S_{k_{0}}$ result in states that are decoupled from each other during parallel transport, effectively reducing the problem of composite bands to $N$ simple bands. If $M$ forms an $N \times N$ matrix whose columns are the eigenvectors of $\Gamma$, we obtain an auxiliary set of parallel transported wavefunctions $\tilde{u}_{n k}$ :

$$
\left|\tilde{u}_{n k}\right\rangle=\sum_{m}^{N} M_{m n}^{\star}\left|u_{m k}^{\|}\right\rangle
$$

where $\gamma_{n} \delta_{m n}=\left(M^{\dagger} \Gamma M\right)_{m n}$. The resulting wave functions $\left\{\left|\tilde{u}_{n k}\right\rangle\right\}$ are independent of the choice of $k_{0}$ and satisfy: $\left\langle\tilde{u}_{m k_{0}}\left|\exp \left(i \frac{2 \pi x}{a}\right)\right| \tilde{u}_{n\left(k_{0}+2 \pi / a\right)}\right\rangle=\exp \left(i \gamma_{n}\right) \delta_{m n}$ where $\gamma_{n}$ is the $n$-th eigen value of $\Gamma$ and $\gamma_{n} \frac{a}{2 \pi}=\langle x\rangle_{n}$.

Wavefunctions $\left\{\left|\tilde{u}_{n k}\right\rangle\right\}$ are not periodic in $k$ with a pe$\operatorname{riod} \frac{2 \pi}{a}$ if $\gamma_{n} \neq 0$. A simple $\mathrm{U}(1)$ transformation can be used to derive $\left\{\left|v_{n k}\right\rangle\right\}$ that have the lattice periodicity in both reciprocal and real space:

$$
\left|v_{n k}\right\rangle=\exp \left\{-i k\left(\frac{\gamma_{n} a}{2 \pi}\right)\right\}\left|\tilde{u}_{n k}\right\rangle
$$


TABLE I: Polarization $\langle x\rangle$ and squared localization length $\ell^{2}$ calculated for a single isolated band $(n=1)$ for different potentials: (1) Gaussian potential with $c=-10.0, d=0.3$; and (2) asymmetric potential with $d_{1}=-0.3, d_{2}=-0.2, c_{1}=$ $-5.0,-c_{2}=-3.0$ both with $a=1.0$ (all in atomic units).

\begin{tabular}{ccccc}
\hline \hline $\mathrm{V}(\mathrm{x})$ & $\langle x\rangle$ & $\ell_{W F}^{2}$ & $\ell_{L R}^{2}$ & $\ell_{B F}^{2}$ \\
\hline$(1)$ & 0.0 & 0.305 & 0.305 & 0.305 \\
$(2)$ & 0.288 & 0.484 & 0.484 & 0.484 \\
\hline \hline
\end{tabular}

In terms of parallel transported wave functions, $\left|v_{n k}\right\rangle=\sum_{m} U_{n m, k}\left|u_{m k}^{\|}\right\rangle$, where

$$
U_{n m, k}=\exp \left\{-i k\left(\frac{\gamma_{n} a}{2 \pi}\right)\right\} M_{m n}^{\star}
$$

In the frame-work of differential geometry [19] the transformation $U_{n m, k}$ projects the $\left\{\left|u_{m k}^{\|}\right\rangle\right\}$in the ray space onto $\left\{\left|v_{n k}\right\rangle\right\}$ in the physical space. Each function $\left|v_{n k}\right\rangle$ is periodic and continuous in $k$-space and can be Fourier transformed to obtain the WF $W_{n}(x, R)$ :

$$
\begin{aligned}
\left|W_{n}(x, R)\right\rangle & =\sum_{k} e^{i k(x-R)}\left|v_{n k}\right\rangle \\
& =\sum_{k, r} e^{i k(x-R)} C_{n r}\left|u_{r k}\right\rangle
\end{aligned}
$$

where $\left\{\left|u_{r k}\right\rangle\right\}$ are the energy eigenfunctions obtained from diagonalization and $C_{n r}=\left\langle v_{n k} \mid u_{r k}\right\rangle$. Eqn (6) is the main formal result of this paper that gives WF in the general case of composite bands for systems that are

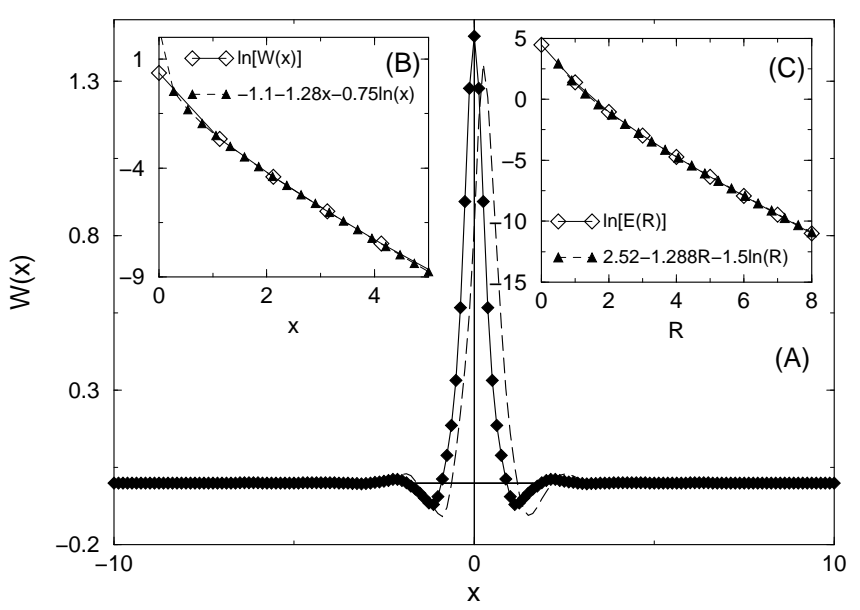

FIG. 1: (A) WF constructed using (6), for the lowest nondegenerate band $(n=1)$ in case of $1 D$ insulator modeled with: Gaussian centro-symmetric potential (solid line) with $c=$ $-10, b=0.3$ and asymmetric potential (dashed line) with $d_{1}=-0.3, d_{2}=-0.2, c_{1}=-5.0,-c_{2}=-3.0$, both with $a=1.0$. (B) and (C) shows decay of WF and $\mathrm{E}(\mathrm{R})$ respectively for the Gaussian centro-symmetric potential. The power law exponents appear as the coefficients of $\ln (\mathrm{x})$ and $\ln (\mathrm{R})$ in $(\mathrm{B})$ and $(\mathrm{C})$ respectively (all in atomic units). periodic in one dimension. Using Eqn (11) and (15), these WFs can be shown to have two important properties (in addition to being orthonormal):

$$
\begin{aligned}
& \text { 1. } \int x W_{n}^{\star}(x, 0) W_{n}(x, 0) d x=\gamma_{n} \\
& \text { 2. } \ell_{W F, n}^{2}=\int x^{2} W_{n}^{\star}(x, 0) W_{n}(x, 0) d x-\gamma_{n}^{2}
\end{aligned}
$$

squared localization length $\ell_{W F, n}^{2}$ is independent of phase factors accompanying the energy eigenfunctions and can be shown to be equal to the one obtained using linear response 20, 21]. Thus, these WFs are the same as maximally localized Wannier functions 13 in 1-dimension. A general form of $U(1)$ transformation in Eqn (4) is $\exp \left\{-i k\left(\frac{\gamma_{n} a}{2 \pi}\right)-i k . L a-i g(k)\right\}$, where $L$ is an integer and $g(k)$ a periodic function of k. It is readily shown that the term $k . L a$ in the exponent alters 11 $<x\rangle_{n}$ by $L a$ but keeps $\ell_{W F, n}^{2}$ unchanged. On the other hand, the term $g(k)$ keeps $\langle x\rangle_{n}$ unchanged, but increases $\ell_{W F, n}^{2}$.

We propose a generalization of expression (6) to metals by including the Fermi-Dirac distribution factor $f\left(E_{n k}, T\right)$ :

$$
\left|W_{n}^{T}(x, R)\right\rangle=\sum_{k, r} e^{i k(x-R)} C_{n r}\left[f\left(E_{r k}, T\right)\right]^{\frac{1}{2}}\left|u_{r k}\right\rangle
$$

These generalized WFs do not form an orthonormal set in general, but yield the same form of density matrix for metals as that for insulators:

$$
\rho^{T}\left(x, x^{\prime}\right)=\sum_{n R} W_{n}^{T \star}(x, R) W_{n}^{T}\left(x^{\prime}, R\right) .
$$

Secondly, they can be shown to have infinite localization length in the case of partially filled bands, arising from the discontinuity in $f\left(E_{n k}, T\right)$ at the Fermi energy [22].

We first use above formalism to construct WFs for an isolated single band in 1D model insulator and metal (partially filled band). We consider two generic types of model potentials: (1) Gaussian centro-symmetric potential: $V(x)=\sum_{n=1}^{\infty} \frac{c}{d \sqrt{2 \pi}} \exp \left[-(x-n a)^{2} / d^{2}\right]$, which was used by $\mathrm{He}$ and Vanderbilt 23. in analysis of spatial decay of WFs and is used to benchmark our calculation, and (2) general asymmetric potential: $V(x)=$ $c_{1}\left[1+\cos \left\{2 \pi\left(x+d_{1}\right) / a\right\}\right]+c_{2}\left[1+\cos \left\{4 \pi\left(x+d_{2}\right) / a\right\}\right]$, which we use to test the universality [23] of decay exponents of WFs. Fig प(A) shows WFs constructed using our formalism for the two potentials.

We calculate the squared localization length $\ell_{n}^{2}$ using three different methods: $\ell_{W F, n}^{2}$ using Eqn (7), $\ell_{L R, n}^{2}=$ $\left\langle\frac{\partial u_{k n}}{\partial k} \mid \frac{\partial u_{k n}}{\partial k}\right\rangle$ obtained using linear response and $\ell_{B F, n}^{2}=$ $\frac{2}{\Delta k^{2}} \sum_{B Z}\left(1-R e\left\langle u_{n, k}^{\|} \mid u_{n, k+\Delta k}^{\|}\right\rangle\right) \triangle k$, calculated from the parallel transported Bloch functions. As summarized in Table \ $\ell^{2}$ calculated using the three methods for both the potentials studied here are identical within numerical errors. As the $\ell_{L R, n}^{2}$ is known to be gauge invariant for 

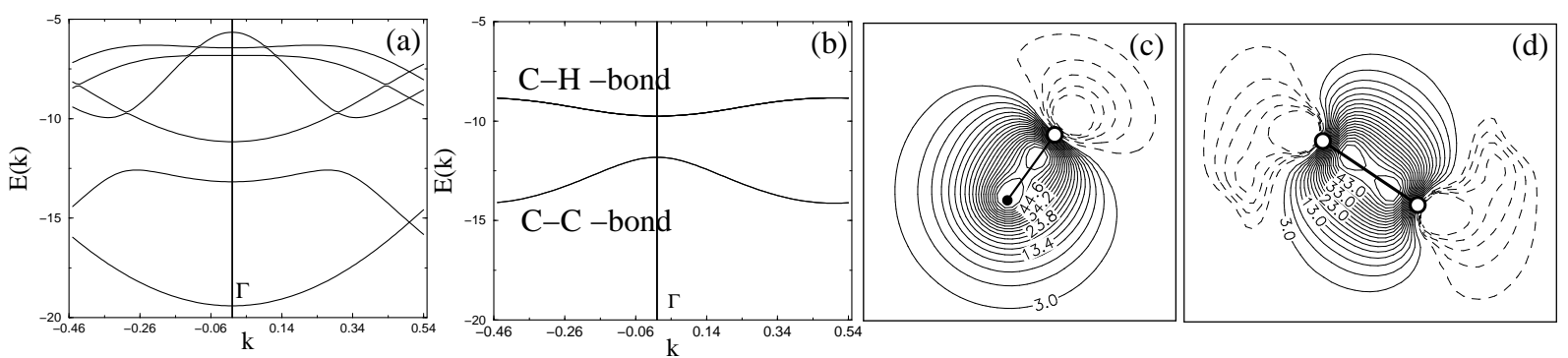

FIG. 2: Polyethylene: (a) Band structure, (b) Transformed band structure of $\left\langle\tilde{u}_{n k}|H(k)| \tilde{u}_{n k}\right\rangle$, (c) WF corresponding to C-H bond, (d) WF corresponding to C-C bond.
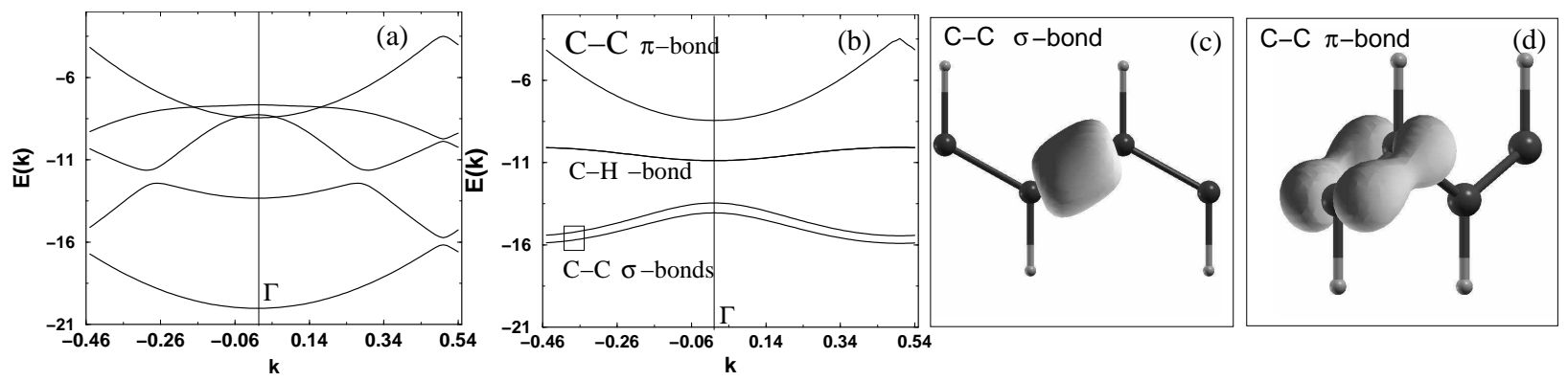

FIG. 3: Polyacetylene: (a) Band structure, (b) Transformed band structure of $\left\langle\tilde{u}_{n k}|H(k)| \tilde{u}_{n k}\right\rangle$, (c) WF corresponding to C-C $\sigma$-bond, (d) WF corresponding to C-C $\pi$-bond. In (c) and (d) the isosurfaces are prepared using XCrysDen software 18].

a given subspace [20], and correspond to maximum localization, this numerically verifies maximal localization of the WFs constructed using the present formalism for the two cases.

We now compare the spatial decay behavior of the present WFs with the maximally localized WFs 13 which exhibit a power law decay 23 in addition to the exponential one 24]: $W_{n}(x) \approx x^{-3 / 4} e^{h x}$, where $h$ is defined from the $k$ - point $\pi / a+i h$ at which $E_{0}=E_{1}$ in the complex band structure. Similarly $E_{n}(R)$, defined as $E_{n}(R)=$ $\left\langle W_{n}(x, R)|H| W_{n}(x, 0)\right\rangle$, decays as: $E(R) \approx x^{-3 / 2} e^{h R}$. The power law decay exponents $-3 / 4$ for $W_{n}(x, R)$ and $-3 / 2$ for $E_{n}(R)$ are universal numbers [23]. Calculating

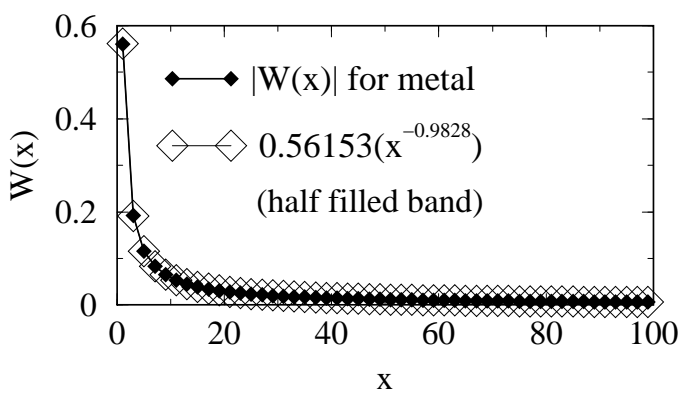

FIG. 4: Decay of WF for metal with half filled band modeled by a centro-symmetric potential at $\mathrm{T}=0 \mathrm{~K}: d 1=0.25, c 1=$ $-5.0, d 2=0, c 2=0$ with $a=1.0$ ( all in atomic units ). $h$ from the complex band structure and fitting these general decay forms to the WFs in this work, we find the same power law exponents for both the model potentials considered. Results for the centro-symmetric potential are shown in Figप(B) and (C).

WF constructed using Eqn. (8) for a partially filled band $(T=0 K)$ is shown in Fig 4 Form of the spatial decay of this WF agrees with that of the density matrix $\left(\propto x^{-\eta}\right)$ predicted analytically by Beigi and Arias 22. We observe that the exponent $\eta$ is between 0.95 and 1 for any fraction of band filling at $0 \mathrm{~K}$. This means the localization length of metals being infinity.

We now illustrate the present method within firstprinciples density functional theory in the general case of composite bands by constructing WFs for two polymer chain molecules: polyethylene (PE) and polyacetylene (PA). A unit cell of a polyethylene (polyacetylene) chain consists of $2\left[\mathrm{CH}_{2}\right](2[\mathrm{CH}])$ with twelve (ten) valence electrons occupying the lowest six (five) bands. The unit cell considered is large enough in the transverse directions to minimize the interaction between the periodic images of the chain. We use standard density functional theory code 25. to carry out these calculations. We used 100 Runge-Kutta steps in the parallel transport and 20 $k$-points in the Fourier transform (Eqn [6]) to obtain WFs.

The electronic structure of PE [Fig[2(a)] apparently groups into two non-crossing sets of bands (the lowest two and the rest). Interestingly, unitary matrix $M$ we 
TABLE II: Polarization $\langle\vec{r}>$ and squared localization length $\ell_{W F}^{2}$ of polyethylene WFs (all lengths are in $\AA$ ).

\begin{tabular}{cccc}
\hline \hline Bond & \multicolumn{2}{c}{$\langle\vec{r}\rangle$} & $\ell_{W F}^{2}$ \\
\hline C-C & $(0.0,0.0,0.0)$ & $(1.25 .0,0.0)$ & 0.24 \\
C-H & $(\mp 0.63,0.53, \pm 0.91)$ & $( \pm 0.63,-0.53, \mp 0.73)$ & 0.23 \\
\hline \hline
\end{tabular}

determined from the geometric phases mixes bands from these groups. We use $M$ to transform the $u_{n k}$ to $\tilde{u}_{n k}$, and plot a transformed band structure [Fig[2(b)] with expectation values of energy of $\tilde{u}_{n k}$. We find two distinct bands: (1) the lower one with double degeneracy corresponding to $\mathrm{C}-\mathrm{C}$ bonds and (2) the upper one with quadruple degeneracy corresponding to $\mathrm{C}-\mathrm{H}$ bonds. In Table III we summarize $\langle\vec{r}\rangle$ and $\ell^{2}$ values for the six WFs in two groups corresponding to the two transformed bands (1) and (2). The average squared localization length $\bar{\ell}_{W F}^{2}=(1 / N) \sum_{n}^{N} \ell_{W F, n}^{2}$ is $0.23 \AA^{2}$ and agrees well with linear response calculation $\bar{\ell}_{L R}^{2}$ of value $0.23 \AA^{2}$ indicating maximal localization. Contour-plots of the two WFs are shown in Fig 2(c,d)

Similarly, electronic structure of PA (Fig 3(a)) yields the transformed band structure (Fig 3 (b)) consisting of four isolated bands: two of them (lowest in energy) are almost degenerate corresponding to the two C-C $\sigma$-bonds of slightly different lengths. The one in the middle is doubly degenerate and corresponds to the two $\mathrm{C}-\mathrm{H}$ bonds and the one highest in energy corresponds to the C-C $\pi$-bond. Fig 3 (c,d) shows isosurfaces of WFs describing C-C $\sigma$ bond $\left(\ell_{W F}^{2}=0.22 \AA^{2}\right)$ and C-C $\pi$ bond $\left(\ell_{W F}^{2}=\right.$ $2.95 \AA^{2}$ ) in polyacetylene. An order of magnitude higher $\ell_{W F}^{2}$ of C-C $\pi$ bonds than the others makes $\pi$-conjugated systems particularly interesting for transport properties.

While the logic behind the present formalism is the construction of simple continuous and periodic functions from parallel transported Bloch functions, the resulting WFs in 1-D are maximally localized. Its generalization to higher dimensions is natural when the geometric phases $\Gamma_{\alpha}$ for paths along different directions in the Brillouin zone commute. In most cases, however, the $\Gamma_{\alpha}$ 's do not commute (corresponding to nontrivial phases for closed paths within the Brillouin zone) implying the WFs can not be perfectly localized in all the three directions simultaneously.

In conclusion, we have presented a formal expression for WFs of 1-d Bloch electrons and a first-principles method for their construction through calculation of geometric phase matrices $\Gamma$. It can be readily implemented in any DFT code that includes linear response. It naturally identifies the groups of bands that make up WFs (or bonds) through the eigenvectors of the $\Gamma$ and their polarization through eigenvalues of $\Gamma$. The method should be very useful in studies of 1-D periodic systems in nano- science.

UVW thanks Karin M. Rabe for many useful discussions. We also thank G. Baskaran, J. Samuel and Ayan Datta for stimulating discussions. JB thanks CSIR, India for a research fellowship. This work was supported by the J Nehru Centre for Advanced Scientific Research, funded by the Department of Science and Technology, Government of India.

[1] G. Wannier, Phys. Rev. 52, 191 (1937).

[2] W. Kohn and J. R. Onffroy, Phys. Rev. B 8, 2485 (1973).

[3] J. Des Cloizeux, Phys. Rev. 129, 554 (1963)

[4] J. Zak, Phys. Rev. Lett. 62, 2747 (1989)

[5] K. M. Rabe and U. V. Waghmare, Phys. Rev. B 52, 13236 (1995).

[6] E. Pavarini, I. Dasgupta, T. Saha-Dasgupta, O. Jepsen, and O. K. Andersen, Phys. Rev. Lett. 87, 047003 (2001)

[7] G. Galli and M. Parrinello, Phys. Rev. Lett. 69, 3574 (1992).

[8] S. Geodecker and L. Colombo, Phys. Rev. Lett. 73, 122 (1994).

[9] M. V. Berry, Proc. R. Soc. London Ser. A 329, 45 (1994)

[10] J. Zak, Europhys. Lett. 9, (7), pp. 615-620 (1989)

[11] R.D. King-Smith and David. Vanderbilt, Phys. Rev. B 47, 1651 (1992).

[12] R. Resta, Berry Phase in Electronic Wave functions, available at: http://ale2ts.ts.infm.it:6163/ resta/publ/notes_trois.ps.gz

[13] N. Marzari and D. Vanderbilt, Phys. Rev. B 56, 12847 (1997).

[14] S. Baroni, P. Giannozzi, and A. Testa, Phys. Rev. Lett. 59, 2662 (1987).

[15] X. Gonze, D. C. Allan, and M. P. Teter, Phys. Rev. Lett. 68, 3603 (1992)

[16] W.H. Press, S.A. Teukolsky, W.T. Vetterling, and B.P. Flannery, Numerical Recipes (Cambridge University Press, Cambridge, England, 1986/1992)

[17] F. Wilczek and A. Zee, Phys. Rev. Lett. 52, 2111 (1984)

[18] A. Kokalj, XCrySDen - a new program for displaying crystalline structures and electron densities, J. Mol. Graphics Modelling, 17, 176 (1999).

[19] J. Samuel and R. Bhandari, Phys. Rev. Lett. 60, 2331 (1988).

[20] M.Viethen, X. Gonze, and Ph. Gosez, Phys. Rev. B 66, (2002).

[21] U. V. Waghmare, N. A. Spaldin, H. C. Kandpal, and R. Seshadri, Phys. Rev. B, 67, 125111 (2003).

[22] S. Ismail-Beigi and T. A. Arias, Phys. Rev. Lett. 82, 2127 (1999).

[23] L. He and D. Vanderbilt, Phys. Rev. Lett. 86, 5341 (2001).

[24] W. Kohn, Phys. Rev. 115, 809 (1959).

[25] CASTEP 2.1, M. C. Payne, X. Weng, B. Hammer, G. Francis, I. Stich, U. Bertram, A. de Vita, J. S. Lin, A. Qteish and V. Milman, Cavendish Laboratory, University of Cambridge; LRESP, U. V. Waghmare, V. Milman and K. M. Rabe. 